\title{
GROWTH OF DISTORTION-PRODUCT OTOACOUSTIC EMISSIONS IN A NONLINEAR, ACTIVE MODEL OF COCHLEAR MECHANICS
}

\author{
S. T. NEELY, M. P. GORGA AND P. A. DORN \\ Boys Town National Research Hospital, 555 North 30 Street, Omaha, Nebraska 68131 \\ E-mail:neely@boystown.org
}

\begin{abstract}
Evidence of compressive growth of basilar membrane displacement can be observed in measurements of DPOAE. When the levels of the two stimulus tones are related by the formula $L_{1}=0.4 L_{2}+39$, then the DPOAE I/O function resembles the classic Fletcher-Munson (1933) loudness function. In order to understand how the DPOAE I/O function relates to the perception of loudness, we need to also understand how each of these response measures relates to basilar membrane displacement. The model of cochlear mechanics described here provides a means to directly compare the rate of growth of these three response measures.
\end{abstract}

\section{Introduction}

The rate of growth of distortion-product otoacoustic emission (DPOAE) level when stimulus levels are optimized [2,3] is remarkably similar to log-loudness [1]. One salient feature in each case is the compressive growth observed at moderate stimulus levels. The compressive growth of basilar membrane (BM) displacement is thought to be the underlying reason for the compressive growth observed in the other two response measures [4]. In this paper, we use a nonlinear, active, twodimensional model of cochlear mechanics to directly compare the rate of growth of DPOAE level, BM displacement, and (simulated) log-loudness in the same ear.

\section{Model}

It has become increasingly clear in recent years that the mechanics of the BM, although highly nonlinear, can often be represented by a linear model of the following form $[5,6,7]$.

$$
Z_{a}=Z_{p}+\gamma Z_{a}
$$

In this equation, $Z_{p}$ is the passive impedance, observed at high stimulus levels or post mortem, $Z_{a}$ is the active impedance that plays a major role at low stimulus levels, $\gamma$ is a parameter that decreases from one at the lowest stimulus level to zero at the highest stimulus level, and $Z_{b}$ is the combined impedance of the basilar membrane (BM). We can implement a time-domain version of the impedance in Eq. (1) by using the following set of differential equations.

moh2002.doc submitted to Tony Gummer on 25-Jul-02 


$$
\begin{gathered}
M_{b} \ddot{\xi}_{b}+R_{b} \dot{\xi}_{b}+K_{b} \xi_{b}+g_{c} R_{c} \dot{\xi}_{c}+g_{c} K_{c} \xi_{c}=-A_{p} P_{f} \\
M_{r} \ddot{\xi}_{r}+R_{r} \dot{\xi}_{r}+K_{r} \xi_{r}-g_{c} R_{c} \dot{\xi}_{c}-g_{c} K_{c} \xi_{c}=-g_{r} V_{m} \\
C_{m} \dot{V}_{m}+G_{m} V_{m}=\gamma g_{f} \xi_{b}
\end{gathered}
$$

Equations (2) - (4) represent the micromechanics of the model at each position along the cochlear partition. Equation (2) describes motion of the BM. Equation (3) describes motion of a second mechanical degree-of-freedom (DOF) that is coupled to the BM. The variable $\xi_{c}$ is the difference between the two DOFs. Equation (4) describes outer hair cell (OHC) motility, which is driven by BM displacement and exerts a force on the second DOF. When all of the coefficients of these differential equations are constants, the ratio of fluid pressure $P_{f}$ to $\mathrm{BM}$ velocity $\xi_{b}$ is proportional to BM impedance $Z_{b}$ and can be expressed in the form of Eq. (1). An electrical-analog equivalent of the micromechanics is shown in Figure (1).

In previous work [9], we have used $\xi_{c}$ as the input the $\mathrm{OHC}$ motility and associated this variable with the shear-displacement between the reticular lamina (RL) and tectorial membrane (TM). We have discovered that this previous formulation of the differential equations produced BM impedance that was incompatible with Eq. (1). We have adopted $\xi_{b}$ as the input to OHC motility in the present work in order to preserve certain intensity-invariant properties that are

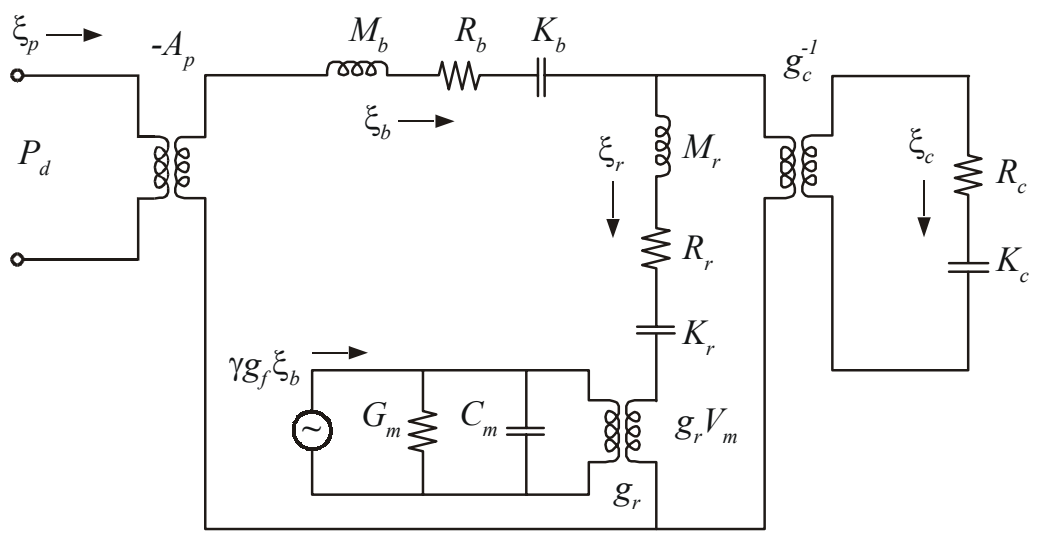

Figure 1. Electrical-analog circuit representing cochlear micromechanics that includes two mechanical resonances and an $\mathrm{OHC}$ feedback force. The electrical current through the $\mathrm{OHC}$ is proportional to $\mathrm{BM}$ displacement at low levels, but saturates at high levels. The OHC feedback force is proportional to voltage across its membrane.

moh2002.doc submitted to Tony Gummer on 25-Jul-02 
associated with Eq. (1) [7]. The physical interpretation of this representation is that the shear displacement, which provides the input to both inner and outer hair cells, is proportional to $\mathrm{BM}$ displacement.

When the coefficient $\gamma$ is a function of BM displacement, the micromechanics become nonlinear. In the present work, we chose the following equation for $\gamma$ to simulate the saturation of $\mathrm{OHC}$ mechano-electric transduction.

$$
\gamma=\gamma_{0}\left(1+\left|\frac{\xi_{b}}{d_{h}}\right|^{\frac{3}{2}}\right)^{-\frac{2}{3}}
$$

Because this is the only nonlinear element in the model, it is directly responsible for both the generation of DPOAEs and the loss of gain (i.e. compression) at high stimulus levels.

One other difference between the present model and previous work is a twodimensional (2-D) representation of the cochlear fluid. Much of our previous work utilized a one-dimensional (1-D) fluid for computational convenience. We discovered that we were able to achieve larger "cochlear amplifier" gains with the 2-D fluid than were possible with the 1-D fluid. In this paper we use 351 points in the longitudinal dimension and 8 points in the other dimension to represent the 2-D cochlear fluid.

\section{Model Results}

Model parameters were chosen to represent salient features of the human cochlea. Consideration was given to the shape and overall sensitivity of BM displacements as well as middle-ear (forward and reverse) transmission. Our best attempt, presented here, still falls short of our goal of achieving a good representation of all aspects of cochlear mechanics.

\subsection{Basilar membrane displacement}

Model results for 1-nm displacement of the BM at four places along the cochlear partition are shown in the Fig. 2A. These tuning curves were derived from impulse responses from a linear, active version of the model, produced by setting $\gamma=1$. The $Q$ values for these tuning curves are typical of neural tuning curves in cat and the group delays (not shown) for the best frequency at each place are approximately equal to estimates derived from SFOAE measurements in human ears [8]. Fig. 2B shows the growth of BM displacement at the 4-kHz place as a function of the sound pressure level of a $4-\mathrm{kHz}$ tone.

moh2002.doc submitted to Tony Gummer on 25-Jul-02 

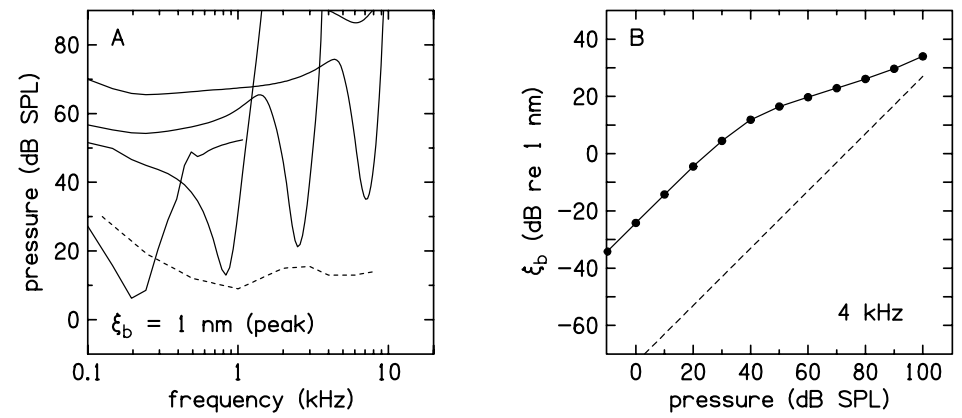

Figure 2. Model results for (A) BM iso-displacement and (B) BM input/output (I/O) function. The dashed line in panel A indicates normal hearing threshold. The dashed line in the panel B is the BM I/O function when $\gamma_{0}=0$.

\subsection{Distortion-product otoacoustic emission}

In Fig. 3, DPOAE levels observed in the model are compared with measurements in human subjects [9]. Stimulus conditions were the same in both cases: $F_{1}=4 \mathrm{kHz}, \mathrm{F}_{2}$ $=3.32 \mathrm{kHz}$, and $\mathrm{L}_{1}=0.4 \cdot \mathrm{L}_{2}+39$. Fig. $3 \mathrm{~A}$ shows DPOAE levels for three groups of human subjects with differing amounts of hearing loss, (1) $20 \mathrm{~dB}$ or less, (2) 25 to $35 \mathrm{~dB}$, and (3) 40 to $50 \mathrm{~dB}$. Note that the DPOAE threshold and rate of growth both increase with hearing loss. In the model, setting the parameter $\gamma_{0}$ to a value less than one simulates hearing loss. Fig. 3B shows the DPOAE level for two model conditions: (1) $\gamma_{0}=1$ and (2) $\gamma_{0}=0.5$. Although, the DPOAE level in the model is roughly the same as in the human ears, the growth rate differs. We will return to this issue later.
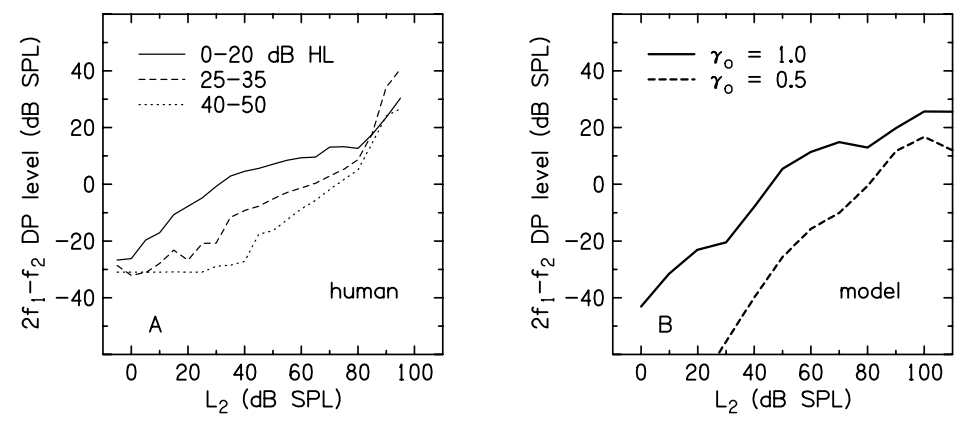

Figure 3. Input-output curves for the $2 \mathrm{~F}_{1}-\mathrm{F}_{2}$ DPOAE as a function of $\mathrm{L}_{2}$ (the level of the higher frequency primary tone). Panel A shows data from three groups of human subjects with varying amounts of hearing loss. Panel B shows model results with and without a simulated mild hearing loss. 


\subsection{Whole-nerve response}

To simulate a whole-nerve (WN) response in the model we apply a soft rectifier to the BM displacement at each position [to represent inner hair cell (IHC) transduction] and sum across the entire length of the BM. In Fig. 4, we compare the model WN response (for a $4-\mathrm{kHz}$ tone) with an empirical estimate of perceptual loudness (for a $1-\mathrm{kHz}$ tone) [1]. An arbitrary scale factor was applied to the WN values to make them similar to the loudness values. The curves in Fig. 4 show the logarithm (base 10) of the WN and loudness values. The good agreement in the slope of the log-WN and log-loudness at low levels (thick and thin solid lines) is a consequence of expansive growth in the IHC soft rectifier. At moderate levels the IHC stage is linear and the BM growth is compressive. Model results for WN are shown for three model conditions: (1) $\gamma_{0}=1$ to simulate normal hearing, (2) $\gamma_{0}=0.5$ to simulate a mild hearing loss (with a threshold shift of about $22 \mathrm{~dB}$ ), and (3) $\gamma_{0}=0$ to simulate a moderate hearing loss (with a threshold shift of about $36 \mathrm{~dB})$.

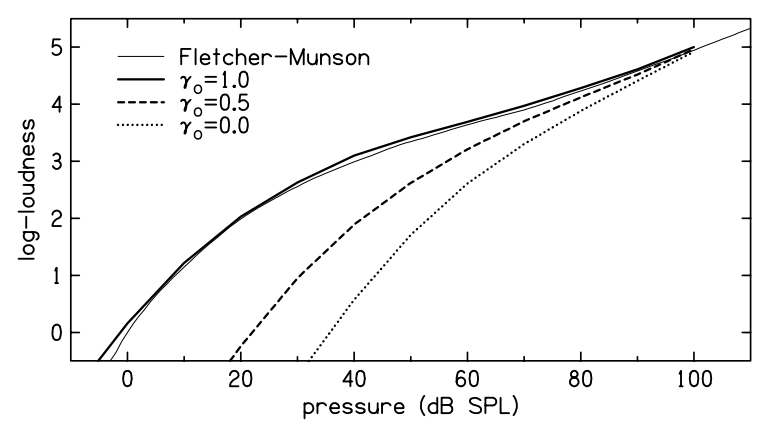

Figure 4. Loudness and WN response as a function of stimulus level. The thin line shows loudness growth based on the measurements of Fletcher and Munson [1]. The thick lines show the model WN response for the normal case and two hearing-impaired cases with threshold shifts of 22 and $36 \mathrm{~dB}$.

\subsection{Compression}

To compare the rates of growth of BM, DPOAE, and WN, we have computed the compression of each of the respective $\mathrm{I} / \mathrm{O}$ curves. We define compression as the reciprocal of the slope of the I/O curve. For the BM and DPOAE I/O curves, compression is the $\mathrm{dB}$ increase in stimulus level divided by the $\mathrm{dB}$ increase in response level. For the $\mathrm{WN}$ curve, we divided the $\mathrm{dB}$ increase in stimulus level by the increase in $10 \cdot \log _{10}$ of the WN value. Figure 5 compares the compression of the model BM, DPOAE and WN responses. Note that the BM growth is linear at low levels when $\gamma_{0}=1$ and linear at all levels when $\gamma_{0}=0$. This can been seen in the slopes of the I/O curves in Fig. $2 \mathrm{~B}$ and is indicated by a compression value of one 

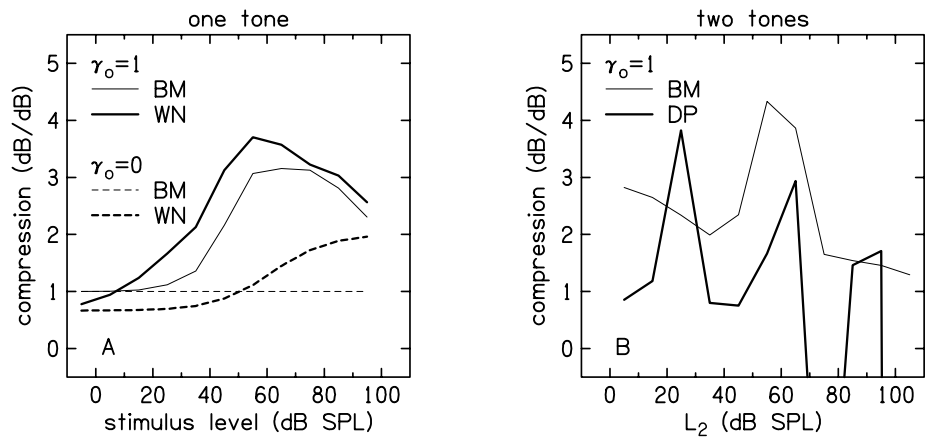

Figure 5. Compression observed in model response measures. Panel A shows the compression of BM and WN growth in response to a single tone (at $4 \mathrm{kHz}$ ) for two model conditions: $\gamma_{0}=1$ and $\gamma_{0}=0$. Panel B shows the compression of BM and WN growth in response to two tones (at 4 and $3.32 \mathrm{kHz}$ ) for $\gamma_{0}=1$. $\mathrm{BM}$ responses are from the $4 \mathrm{kHz}$ place.

in Fig. 5A. The compression of the WN response is less than one at $0 \mathrm{~dB}$ SPL due to the expansive non-linearity of the IHC soft-rectifier. In the case of moderate hearing loss $\left(\gamma_{0}=0\right)$, the region of expansive growth is extended to about $50 \mathrm{~dB}$ SPL. The rate of growth of the WN response is approximately the same at threshold for differing amounts of hearing loss because it is mostly determined by the properties of IHC transduction. Fig. 5B shows the compression of the BM and DPOAE responses when the stimulus consists of two tones. The monotonic results are undesirable and may be partly due to mismatched impedance at the stapes boundary of the cochlear model that causes excessive basal reflection.

\section{Discussion}

The cochlear model described here is sufficiently comprehensive to simulate BM, DPOAE, and WN responses simultaneously. In the current version of the model, the single tone responses appear to exhibit realistic response growth as observed in the $\mathrm{BM}$ and $\mathrm{WN}$ responses. However, the BM and DPOAE responses to two-tone stimuli do not yet appear to be realistic and require further investigation. The observation in the model that the rate of $\mathrm{WN}$ growth is nearly independent of amount of hearing loss is consistent with recent measures of the rate of growth of loudness in normal and hearing-impaired subjects [10]. This result is a direct consequence of linear BM growth at threshold, regardless of the amount of hearing loss.

moh2002.doc submitted to Tony Gummer on 25-Jul-02 


\section{Acknowledgments}

Supported by a grant (DC02251) from the National Institutes of Health.

\section{References}

1. Fletcher, H. and Munson (1933). Loudness, its definition, measurement, and calculation. J. Acoust. Soc. Am., 5, 82-108.

2. Kummer, P., Janssen, T., Hulin, P., and Arnold, W. (2000). Optimal L(1)-L(2) primary tone level separation remains independent of test frequency in humans. Hear Res. 146, 47-56. .

3. Dorn, P. A., Konrad-Martin, D., Neely, S. T., Keefe, D. H., Cyr, E., and Gorga, M. P. (2001). Distortion product otoacoustic emission input/output functions in normal-hearing and hearing-impaired human ears. J Acoust Soc Am. 110, 31193131.

4. Yates, G. K. (1990). Basilar membrane nonlinearity and its influence on auditory nerve rate-intensity functions. Hear. Res., 50, 145-162.

5. Neely, S.T. (1983). The cochlear amplifier. In: E. de Boer, \& M.A. Viergever (Eds.), Mechanics of Hearing. Delft University Press, Delft, The Netherlands, pp 111-118.

6. de Boer, E. and Nuttall, A. L. (2002). The mechanical waveform of the basilar membrane. IV. Tone and noise stimuli. J Acoust Soc Am. 111, 979-989.

7. Shera, C. A. (2001). Intensity-invariance of fine time structure in basilarmembrane click responses: implications for cochlear mechanics. J Acoust Soc Am. 110, 332-348.

8. Shera, C. A., Guinan, J. J., and Oxenham, A. J. (2002). Revised estimates of human cochlear tuning from otoacoustic and behavioral measurements. Proc. Natl. Acad. Sci. 99, 3318-3323.

9. Neely, S. T., Gorga, M. P., and Dorn, P. A. (1993). Distortion Product and Loudness Growth in an Active, Nonlinear Model of Cochlear Mechanics. In: H. Wada, T. Takasaka. K. Ikeda, K. Ohyama, \& T. Koike (Eds.), Recent Developments in Auditory Mechanics. World Scientific, Singapore, pp. 237243.

10. Buus, S. and Florentine, M. (2001). Growth of Loudness in Listeners with Cochlear Hearing Losses: Recruitment Reconsidered. J. Assoc. Res. Otolaryn. 3, 120-139. 